\title{
BROWN REPRESENTABILITY AND THE EILENBERG-WATTS THEOREM IN HOMOTOPICAL ALGEBRA
}

\author{
MARK HOVEY \\ (Communicated by Michael A. Mandell)
}

\begin{abstract}
It is well known that every homology functor on the stable homotopy category is representable, so of the form $E_{*}(X)=\pi_{*}(E \wedge X)$ for some spectrum E. However, Christensen, Keller, and Neeman (2001) have exhibited simple triangulated categories, such as the derived category of $k[x, y]$ for sufficiently large fields $k$, for which not every homology functor is representable. In this paper, we show that this failure of Brown representability does not happen on the model category level. That is, we show that a homology theory is representable if and only if it lifts to a well-behaved functor on the model category level. We also show that, for a reasonable model category $\mathcal{M}$, every functor that has the same formal properties as a functor of the form $X \mapsto X \otimes E$ for some cofibrant $E$ is naturally weakly equivalent to a functor of that form. This is closely related to the Eilenberg-Watts theorem in algebra, which proves that every functor with the same formal properties as the tensor product with a fixed object is isomorphic to such a functor.
\end{abstract}

\section{INTRODUCTION}

Triangulated categories abound in modern mathematics, particularly in algebraic geometry and algebraic topology. Triangulated categories are additive categories $\mathcal{T}$ with an endofunctor $\Sigma: \mathcal{T} \rightarrow \mathcal{T}$ that is an equivalence of categories and a collection of exact triangles of the form

$$
A \stackrel{f}{\rightarrow} B \rightarrow C \rightarrow \Sigma A
$$

satifying various properties. One can think of a triangulated category as like an abelian category except there are only long exact sequences, not short exact sequences. In particular, there are no kernels and cokernels, only cofibers which mix them together; in the above diagram $C$ is the cofiber of $f$. The stable homotopy category is the prototypical triangulated category, but the derived category of a ring or scheme is also one.

Triangulated categories are typically studied by exact functors to abelian categories, where $F: \mathcal{T} \rightarrow \mathcal{A}$ is exact if it takes exact triangles in $\mathcal{T}$ to exact sequences in the abelian category $\mathcal{A}$. Such a functor that also preserves coproducts is referred to as a homology functor. (A homology functor to $\mathcal{A}^{\text {op }}$ is a cohomology functor on $\mathcal{T}$.) If $\mathcal{T}$ is closed symmetric monoidal with unit $S$, then for any object $E$ of $\mathcal{T}$, the functor

$$
E_{0} X=\mathcal{T}(S, X \otimes E)
$$

\footnotetext{
Received by the editors September 24, 2013 and, in revised form, December 10, 2013.

2010 Mathematics Subject Classification. Primary 55U35; Secondary 18E30, 18G35, 55N20, $55 \mathrm{P} 42$.
} 
is a homology functor. So it is natural to wonder if these are the only homology functors to abelian groups; if so we say that Brown representability for homology holds in $\mathcal{T}$. This is true in the stable homotopy category and in the derived category $\mathcal{D}(R)$ of a countable commutative ring $R$. But it is false in general, as explained in CKN01. Brown representability for cohomology functors, on the other hand, holds under quite weak hypotheses on $\mathcal{T}$.

Now, in practice, triangulated categories tend to come from more highly structured categories, such as stable model categories [Hov99, Chapter 7] or stable infinity-categories. The basic thesis of this paper is that the homological functors that arise from these more structured categories are precisely the ones that satisfy Brown representability. We choose to work in model categories as opposed to infinity-categories, but there are versions of this theorem for infinity-categories as well.

We describe a typical theorem along these lines. For this, let $\mathrm{Ch}(R)$ denote the category of unbounded chain complexes of right $R$-modules for a fixed commutative ring $R$ with 1 ; we assume $R$ is commutative in order to have an internal tensor product (otherwise we would have to modify the definition of representable). We give this the projective model structure, where fibrations are surjections and weak equivalences are homology isomorphisms. Following [Kel94], a functor $G$ : $\mathrm{Ch}(R) \rightarrow$ $\mathrm{Ch}(S)$ is called a DG-functor if it is enriched over $\mathrm{Ch}(\mathbb{Z})$; that is, if there are natural chain maps $\operatorname{Hom}_{R}(X, Y) \rightarrow \operatorname{Hom}_{S}(G X, G Y)$ of Hom complexes that are compatible with compositions and identities. Recall that $\operatorname{Hom}_{R}(X, Y)$ is the chain complex of abelian groups whose entry in degree $n$ is $\bigoplus_{i} \operatorname{Hom}_{R}\left(X_{i}, Y_{n+i}\right)$ with the usual differential, so that cycles are chain maps. The functoriality of $G$ is recovered by looking at the map of Hom complexes applied to the cycles in degree 0 .

Theorem 0.1. Let $R$ be a commutative ring. A homology functor $F: \mathcal{D}(R) \rightarrow A b$ is representable if and only if there is a left Quillen DG-functor $G: C h(R) \rightarrow C h(R)$ and a natural isomorphism from $H_{0}(L G)$ to $F$, where $L G$ is the total left derived functor of $G$.

As stated, a version of this result could be recovered from Keller's work on DGcategories [Kel94]. The power of the methods in this paper is that these theorems apply much more generally (see Corollary 1.2). For example, we also get a version of this theorem for spectra. Let $S p^{\Sigma}$ denote the model category of symmetric spectra as in [HSS00]. This too is a symmetric monoidal category, so there is a notion of a symmetric ring spectrum $R$ and of right modules over $R$. The category $R$-Mod of right modules is not abelian, but it inherits a model structure whose homotopy category $\mathcal{D}(R)$ is triangulated, so can be thought of as the derived category of $R$. There is also a notion of a spectral functor analogous to that of a DG-functor.

Theorem 0.2. Let $R$ be a symmetric commutative ring spectrum. A homology functor $F: \mathcal{D}(R) \rightarrow A b$ is representable if and only if there is a left Quillen spectral functor $G: R$-Mod $\rightarrow R$-Mod and a natural isomorphism from $\pi_{0}(L G)$ to $F$, where $L G$ is the total left derived functor of $G$.

These theorems are homological versions of internal Eilenberg-Watts theorems that characterize the tensor product (and do not require commutativity). For these theorems, if $R$ is a monoid in a model category, we give the category $R$-Mod of right $R$-modules the model structure in which the weak equivalences (resp. fibrations) are maps of $R$-modules that are weak equivalences (resp. fibrations) in the underlying 
model category. This model structure will always exist for the model categories we consider. We also use the letter $Q$ to denote cofibrant replacement in the relevant model category.

Theorem 0.3. Let $R$ and $R^{\prime}$ be differential graded algebras, and let $G: R$-Mod $\rightarrow$ $R^{\prime}$-Mod be a left Quillen DG-functor. Then there is a natural isomorphism

$$
Q X \otimes_{R} G R \rightarrow(L G)(X)
$$

of functors on Ho $R$-Mod, where $G R$ is a left $R$-module through the map of differential graded algebras

$$
R=\operatorname{Hom}_{R}(R, R) \stackrel{G}{\rightarrow} \operatorname{Hom}_{R^{\prime}}(G R, G R) .
$$

Similarly, if $G: R$-Mod $\rightarrow\left(R^{\prime} \text {-Mod }\right)^{\text {op }}$ is a contravariant left Quillen DG-functor, then there is a natural isomorphism

$$
(L G)(X) \rightarrow \operatorname{Hom}_{R}(Q X, G R)
$$

of functors on Ho $R$-Mod.

Again, this theorem (and stronger versions of it that take into account special features of chain complexes) can be derived from Keller's work Kel94]. The strength of our approach is that it applies to more general situations. The spectral version of Theorem 0.3 is the following.

Theorem 0.4. Let $R$ and $R^{\prime}$ be symmetric ring spectra, and let $G: R$-Mod $\rightarrow$ $R^{\prime}$-Mod be a left Quillen spectral functor. Then there is a natural isomorphism

$$
Q X \otimes_{R} G R \rightarrow(L G)(X)
$$

of functors on Ho $R$-Mod, where $G R$ is a left $R$-module through the map of symmetric ring spectra

$$
R=\operatorname{Hom}_{R}(R, R) \stackrel{G}{\longrightarrow} \operatorname{Hom}_{R^{\prime}}(G R, G R) .
$$

Similarly, if $G: R$-Mod $\rightarrow\left(R^{\prime} \text {-Mod }\right)^{\text {op }}$ is a contravariant left Quillen spectral functor, then there is a natural isomorphism

$$
(L G)(X) \rightarrow \operatorname{Hom}_{R}(Q X, F R)
$$

of functors on $\mathrm{Ho}-\mathrm{Mod} R$.

Of course, both Theorem 0.3 and Theorem 0.4 are special cases of a more general theorem about model categories, Theorem 1.1.

The work for this paper was done in 2009 and the author apologizes for the publication delay. The author owes a debt of thanks to his former student Manny Lopez, who first introduced him to the Eilenberg-Watts theorem. He also wishes to express his debt to Dan Christensen, Bernhard Keller, and Amnon Neeman, both for writing the paper CKN01 and for their comments on an early draft. 


\section{EILENBERG-WATtS FOR MODEL CATEGORIES}

Our goal is to prove our main theorem and the corollary immediately following it.

Theorem 1.1. Suppose $\mathcal{M}$ is a strongly cofibrantly generated, stable, monogenic, symmetric monoidal model category where the unit is cofibrant. Let $R$ and $R^{\prime}$ be monoids in $\mathcal{M}$, and $G: R$-Mod $\rightarrow R^{\prime}$-Mod be a left Quillen $\mathcal{M}$-functor. Then there is a natural isomorphism

$$
Q X \otimes_{R} G R \rightarrow G Q X=(L G)(X)
$$

of functors on Ho $R$-Mod. Similarly, if $G: R$-Mod $\rightarrow\left(R^{\prime} \text {-Mod }\right)^{\text {op }}$ is a contravariant left Quillen $\mathcal{M}$-functor, then there is a natural isomorphism

$$
(L G)(X)=G Q X \rightarrow \operatorname{Hom}_{R}(Q X, F R)
$$

of functors on Ho R-Mod.

Corollary 1.2. Suppose $R$ is a commutative monoid in a strongly cofibrantly generated, stable, monogenic, symmetric monoidal model category $\mathcal{M}$ where the unit $S$ is cofibrant. Then a homology functor $F$ : Ho $R$-Mod $\rightarrow A b$ is representable if and only if there is a left Quillen $\mathcal{M}$-functor $G: R$-Mod $\rightarrow R$-Mod and a natural isomorphism from $\pi_{0}(L G)$ to $F$, where $L G$ is the total left derived functor of $G$ and $\pi_{0}$ is the functor $\operatorname{Ho} R-\operatorname{Mod}(R,-)$.

Even without defining the terms in Theorem 1.1. we can use it to derive Corollary 1.2 .

Proof. Suppose $F$ is representable, so that we have a natural isomorphism

$$
E_{0} X=\operatorname{Ho} R-\operatorname{Mod}\left(R, X \otimes_{R}^{L} E\right) \cong F X
$$

for some object $E$ in Ho $R$-Mod, where the superscript on the tensor indicates the left derived functor of tensor (which is the internal tensor in Ho $R$-Mod). We may as well assume that $E$ is cofibrant in $R$-Mod, in which case the functor $G X=X \otimes_{R} E$ is a left Quillen $\mathcal{M}$-functor on $R$-Mod such that $\pi_{0}(L G)$ is naturally isomorphic to $F$.

Conversely, if there is a lift $G: R$-Mod $\rightarrow R$-Mod as in the corollary, then Theorem 1.1 gives us a natural isomorphism

$$
X \otimes_{R}^{L} G R=Q X \otimes_{R} G R \rightarrow(L G)(X),
$$

and so a natural isomorphism

$$
(G R)_{0} X=\operatorname{Ho} R-\operatorname{Mod}\left(R, X \otimes_{R}^{L} G R\right) \rightarrow F X,
$$

showing that $F$ is representable.

Note that one can modify representability for homology functors $F$ on modules over a non-commutative monoid $R$ by requiring

$$
F X \cong \mathcal{M}\left(S, X \otimes_{R}^{L} E\right)
$$

for some left $R$-module $E$. Then a suitably modified version of Corollary 1.2 will work with the same proof.

We will now define the terms in Theorem 1.1. Obviously some background in model categories is required; standard sources include Hir03 and Hov99, in addition to the essential paper of Schwede and Shipley [SS00]. Symmetric monoidal 
model categories are defined in [Hov99, Chapter 4]. In particular, they are automatically closed symmetric monoidal categories. Thus, if $\mathcal{M}$ is a symmetric monoidal model category and $R$ is a monoid in $\mathcal{M}, R$-Mod is tensored, cotensored, and enriched over $\mathcal{M}$. So we can define $\mathcal{M}$-functors in analogy with DG-functors and spectral functors. This is part of the general theory of enriched categories and functors.

Stable model categories are defined in Hov99, Chapter 7]. A stable symmetric monoidal model category $\mathcal{M}$ is monogenic if the unit $S$ is a compact weak generator of the triangulated category $\operatorname{Ho} \mathcal{M}$. This means that the functor

$$
\operatorname{Ho} \mathcal{M}(S,-)_{*}
$$

from Ho $\mathcal{M}$ to graded abelian groups commutes with all coproducts and reflects 0 , so that $\operatorname{Ho} \mathcal{M}(S, X)_{*}=0$ implies that $X \cong 0$ in $\operatorname{Ho} \mathcal{M}$.

This leaves us with defining strongly cofibrantly generated.

Definition 1.3. Suppose $\mathcal{M}$ is a symmetric monoidal model category. We say that $\mathcal{M}$ is strongly cofibrantly generated if there are sets $I$ of cofibrations in $\mathcal{M}$ and $J$ of trivial cofibrations in $\mathcal{M}$ such that, for every monoid $R$ in $\mathcal{M}$, the sets $I \otimes R$ and $J \otimes R$ cofibrantly generate a model structure on $R$-Mod where the weak equivalences are maps of $R$-modules that are weak equivalences in $\mathcal{M}$.

The reader might wonder why we make this definition, instead of simply assuming that $\mathcal{M}$ satisfies the monoid axiom of [SS00]. The answer is that the paper [SS00] assumes that all objects of $\mathcal{M}$ are small with respect to the entire category $\mathcal{M}$, and we prefer not to make that assumption.

Note that this definition implies that the maps of $I \otimes R$ are small with respect to $(I \otimes R)$-cell, and similarly for $J \otimes R$, as this is part of the definition of cofibrantly generated. Note also that a map $p$ is a fibration (resp. trivial fibration) in $R$-Mod if and only if it has the right lifting property with respect to the maps of $J \otimes R$ (resp. $I \otimes R$ ), which is equivalent to $p$ being a fibration (resp. trivial fibration) in $\mathcal{M}$. This definition also implies that the functor that takes $X \in \mathcal{M}$ to $X \otimes R \in R$-Mod is a left Quillen functor.

As noted above, the work of SS00 implies the following theorem.

Theorem 1.4 (Schwede-Shipley). Suppose $\mathcal{M}$ is a cofibrantly generated, closed symmetric monoidal model category. If $\mathcal{M}$ satisfies the monoid axiom of [SS00, Definition 3.3] and every object of $\mathcal{M}$ is small, then $\mathcal{M}$ is strongly cofibrantly generated.

An example of a strongly cofibrantly generated model category in which not every object is small is the category of (compactly generated weak Hausdorff) topological spaces.

Now Theorem 0.3 and Theorem 0.4 are immediate corollaries of Theorem 1.1 , and thus Theorem 0.1 and Theorem 0.2 follow immediately from Corollary 1.2 , For Theorem 0.3 the base model category $\mathcal{M}$ is the category $\mathrm{Ch}(\mathbb{Z})$ in the projective model structure, in which the weak equivalences are the quasi-isomorphisms and the fibrations are the surjections. This model structure is well known to be stable, symmetric monoidal, and monogenic with cofibrant unit. It is strongly cofibrantly generated by [S00, Section 5]. A DG-functor is the same thing as an $\mathcal{M}$-functor, and a monoid in $\mathrm{Ch}(\mathbb{Z})$ is of course a differential graded algebra. Thus Theorem 0.3 follows immediately. Theorem 0.4 is even simpler, because the paper SS00] was written precisely to show that symmetric spectra are strongly cofibrantly generated. 
We now prove Theorem 1.1 in a series of propositions.

Proposition 1.5. Suppose $\mathcal{M}$ is a closed symmetric monoidal category, $R$ and $R^{\prime}$ are monoids in $\mathcal{M}$, and

$$
G: R \text {-Mod } \rightarrow R^{\prime} \text {-Mod }
$$

is an $\mathcal{M}$-functor. Then $G R$ is an $R$ - $R^{\prime}$-bimodule, and there is a natural transformation

$$
\tau: X \otimes_{R} G R \rightarrow G X .
$$

of $R^{\prime}$-modules that is an isomorphism when $X=R$. Similarly, if $G$ is a contravariant $\mathcal{M}$-functor, then $G R$ is an $R \otimes R^{\prime}$-module, and there is a natural transformation

$$
\tau: G X \rightarrow \operatorname{Hom}_{R}(X, G R)
$$

of $R^{\prime}$-modules that is an isomorphism when $X=R$.

Proof. We begin with the covariant case. We will use $\operatorname{Hom}(-,-)$ for the internal Hom functor in $\mathcal{M}$, and $\operatorname{Hom}_{R}(-,-)$ for the enrichment of $R$-Mod in $\mathcal{M}$. For any monoid $R$, we have an isomorphism of monoids

$$
R \cong \operatorname{Hom}(S, R) \cong \operatorname{Hom}_{R}(R, R) .
$$

Because $G$ is an $\mathcal{M}$-functor, there is an induced map

$$
\operatorname{Hom}_{R}(R, R) \rightarrow \operatorname{Hom}_{R^{\prime}}(G R, G R)
$$

of monoids. The map of right $R^{\prime}$-modules

$$
R \otimes G R \rightarrow G R
$$

adjoint to the map

$$
R \cong \operatorname{Hom}_{R}(R, R) \stackrel{G}{\rightarrow} \operatorname{Hom}_{R^{\prime}}(G R, G R)
$$

makes $G R$ an $R$ - $R^{\prime}$-bimodule, though there are many details for the conscientious reader to check.

Similarly, for $X \in R$-Mod, the composite

$$
X \stackrel{\cong}{\rightarrow} \operatorname{Hom}_{R}(R, X) \rightarrow \operatorname{Hom}_{R^{\prime}}(G R, G X)
$$

has adjoint the desired natural transformation

$$
\tau: X \otimes_{R} G R \rightarrow G X
$$

of $R^{\prime}$-modules. There are even more details to check here. In particular, a priori the adjoint to

$$
\phi_{X}: X \rightarrow \operatorname{Hom}_{R^{\prime}}(G R, G X)
$$

is just a map

$$
X \otimes G R \rightarrow G X
$$

of right $R^{\prime}$-modules. However, $\phi_{X}$ is in fact a map of right $R$-modules, using the left $R$-module structure on $G R$ to make the target of $\phi_{X}$ a right $R$-module. This means that the adjoint descends through the relevant coequalizer diagram to give the desired map

$$
\tau: X \otimes_{R} G R \rightarrow G X .
$$

The contraviariant case is similar. In this case, we get the right $R$-module structure on the $R^{\prime}$-module $G R$ via the adjoint to the map of monoids

$$
R^{\mathrm{op}} \stackrel{\cong}{\rightarrow} \operatorname{Hom}_{R}(R, R)^{\mathrm{op}} \rightarrow \operatorname{Hom}_{R^{\prime}}(G R, G R) .
$$


Here, if $T$ is a monoid, $T^{\mathrm{op}}$ is the monoid with the reversed multiplication. Similarly, for $X \in R$-Mod, we have the map

$$
X \cong \operatorname{Hom}_{R}(R, X) \rightarrow \operatorname{Hom}_{R^{\prime}}(G X, G R),
$$

which is in fact a map of right $R$-modules. This has adjoint a map

$$
G X \rightarrow \operatorname{Hom}(X, G R),
$$

which in fact factors through $\operatorname{Hom}_{R}(X, G R)$, giving us the desired natural transformation.

Now suppose that $\mathcal{M}$ is a strongly cofibrantly generated symmetric monoidal model category where the unit is cofibrant, $R$ and $R^{\prime}$ are monoids in $\mathcal{M}$, and $G: R$-Mod $\rightarrow R^{\prime}$-Mod is a left Quillen $\mathcal{M}$-functor. Note that the strongly cofibrantly generated hypothesis guarantees that $R$-Mod and $R^{\prime}$-Mod inherit model structures from $\mathcal{M}$, so this makes sense. Then $R$ is cofibrant in $R$-Mod, so $G R$ is cofibrant as an $R^{\prime}$-module. However, at first glance one would not expect this to be enough to conclude that the functor $H: R$-Mod $\rightarrow R^{\prime}$-Mod defined by

$$
H X=X \otimes_{R} G R
$$

to be left Quillen. So our next goal is to prove this.

We first point out that our hypotheses on $\mathcal{M}$ guarantee that $R$-Mod is an $\mathcal{M}$ model category, in the following sense.

Lemma 1.6. Suppose $\mathcal{M}$ is a strongly cofibrantly generated, closed symmetric monoidal model category, and $R$ is a monoid in $\mathcal{M}$. If $f: A \rightarrow B$ is a cofibration in $\mathcal{M}$ and $g: M \rightarrow N$ is a cofibration in $R$-Mod, then the pushout product

$$
f \square g:(B \otimes M) \amalg_{A \otimes M}(A \otimes N) \rightarrow B \otimes N
$$

is a cofibration in $R$-Mod, which is a trivial cofibration if either $f$ or $g$ is so. In particular, if $M$ is cofibrant in $R$-Mod, and $f$ is a cofibration in $\mathcal{M}$, then $f \otimes M$ is a cofibration in $R$-Mod, and is a trivial cofibration if $f$ is so.

Proof. The last sentence follows from the rest of the proposition by taking $g$ to be the map $0 \rightarrow M$. It suffices to check the statement about $f \square g$ when $f$ and $g$ are generating cofibrations or trivial cofibrations Hov99, Corollary 4.2.5]. In this case, $g$ will be of the form $h \otimes R$ for a map $h$ in either $I$ or $J$, and $f$ will be in either $I$ or $J$. But then

$$
f \square(h \otimes R) \cong(f \square h) \otimes R
$$

so the result follows from the fact that $\mathcal{M}$ is a monoidal model category and tensoring with $R$ is a left Quillen functor from $\mathcal{M}$ to $R$-Mod.

We can now prove that

$$
H X=X \otimes_{R} G R
$$

is a left Quillen functor.

Proposition 1.7. Suppose $\mathcal{M}$ is a strongly cofibrantly generated, closed symmetric monoidal model category, $R$ and $R^{\prime}$ are monoids in $\mathcal{M}$, and $M$ is an $R$ - $R^{\prime}$-bimodule that is cofibrant as a right $R^{\prime}$-module. Then the functor

$$
H X=X \otimes_{R} M: R-\operatorname{Mod} \rightarrow R^{\prime}-\operatorname{Mod}
$$


is a left Quillen functor, with right adjoint $Y \mapsto \operatorname{Hom}_{R^{\prime}}(M, Y)$. Similarly, if $N$ is an $R \otimes R^{\prime}$-module that is fibrant as an object of $\mathcal{M}$, then the functor

$$
H X=\operatorname{Hom}_{R}(X, N): R \text {-Mod } \rightarrow R^{\prime} \text {-Mod }
$$

is a contravariant left Quillen functor, with right adjoint $Y \mapsto \operatorname{Hom}_{R^{\prime}}(Y, N)$.

Proof. We begin with the covariant case, and leave to the reader the check that $\operatorname{Hom}_{R^{\prime}}(M,-)$ is indeed right adjoint to $H$. Note that we use the left $R$-module structure on $M$ to make $\operatorname{Hom}_{R^{\prime}}(M,-)$ into a right $R$-module.

We need to show that if $f$ is a cofibration or trivial cofibration in $R$-Mod, then $f \otimes_{R} M$ is a cofibration or trivial cofibration in $R^{\prime}$-Mod. The proof is similar in both cases, so we just work with cofibrations. Let $I$ be a set of generating cofibrations in $\mathcal{M}$ so that $I \otimes R$ is a set of generating cofibrations in $R$-Mod. Then any cofibration in $R$-Mod is a retract of a transfinite composition of pushouts of maps of $I \otimes R$. Since the tensor product is a left adjoint, it preserves retracts (of course), transfinite compositions, and pushouts. So it suffices to show that if $f \in I$, then

$$
(f \otimes R) \otimes_{R} M
$$

is a cofibration in $R^{\prime}$-Mod. But of course

$$
(f \otimes R) \otimes_{R} M \cong f \otimes M,
$$

so because $M$ is cofibrant as a right $R^{\prime}$-module, Lemma 1.6 completes the proof.

The contravariant case is mostly similar. Again, we leave to the reader the proof of the adjointness relation, where one must take into account the fact that the functors are contravariant. We must then show that if $f$ is a cofibration (resp. trivial cofibration) in $R$-Mod, then $\operatorname{Hom}_{R}(f, N)$ is a fibration (resp. trivial fibration) in $R^{\prime}$-Mod, or equivalently, in $\mathcal{M}$. As before, the two cases are similar, so we only do the cofibration case. Again, every cofibration in $R$-Mod is a retract of a transfinite composition of pushouts of maps of $I \otimes R$, where $I$ is a set of generating cofibrations of $\mathcal{M}$. Since $\operatorname{Hom}_{R}(-, N)$ is a contravariant left adjoint, it preserves retracts, converts transfinite compositions to inverse transfinite compositions, and converts pushouts to pullbacks. Since retracts, inverse transfinite compositions, and pullbacks of fibrations are fibrations, it suffices to check that

$$
\operatorname{Hom}_{R}(g \otimes R, N)=\operatorname{Hom}(g, N)
$$

is a fibration for all $g \in I$. But this is true because $N$ is fibrant in $\mathcal{M}$.

So at this point in the proof of Theorem 1.1 for the covariant case, we have constructed a natural transformation

$$
X \otimes_{R} G R \rightarrow G X
$$

of left Quillen $\mathcal{M}$-functors that is an isomorphism when $X=R$. This induces a natural transformation of the total left derived functors

$$
\phi_{X}: Q X \otimes_{R} G R \rightarrow G Q X=(L G)(X),
$$

that is an isomorphism when $X=R$.

Thus far, we have not used the fact that $\mathcal{M}$ is stable and mongenic.

Lemma 1.8. Suppose $\mathcal{M}$ is a strongly cofibrantly generated, stable, monogenic, symmetric monoidal model category where the unit is cofibrant. Let $R$ be a monoid in $\mathcal{M}$. Then $R$-Mod is also a stable model category. If $\mathcal{M}$ is monogenic, then $R$-Mod is also monogenic, with $R$ as a compact weak generator of Ho $R$-Mod. 
Proof. The homotopy category Ho $R$-Mod is tensored over Ho $\mathcal{M}$. Let $S^{1} \in$ Ho $\mathcal{M}$ denote the suspension of the unit $S$. Then tensoring with $S^{1}$ gives the suspension in Ho $R$-Mod. Because $\mathcal{M}$ is stable, there is an element $S^{-1} \in$ Ho $\mathcal{M}$ such that $S^{-1} \otimes^{L} S^{1} \cong S$. But then tensoring with $S^{-1}$ gives an inverse to the suspension in Ho $R$-Mod, so $R$-Mod is stable.

Now consider the adjunction $\mathcal{M} \rightarrow R$-Mod whose left adjoint is given by tensoring with $R$ and whose right adjoint is the forgetful functor $U$. This induces a corresponding adjunction Ho $\mathcal{M} \rightarrow$ Ho $R$-Mod whose right adjoint is the total right derived functor $U_{r}$ of $U$. However, by definition of the model structure on $R$-Mod, $U$ preserves all weak equivalences. Thus $U_{r}(X) \cong U X$ for all $X$. It then follows easily that $U_{r}$ preserves coproducts and reflects 0 , in the sense that $U_{r} X \cong 0$ if and only if $X \cong 0$.

Now the isomorphism

$$
\operatorname{Ho} R-\operatorname{Mod}(R, X) \cong \operatorname{Ho} \mathcal{M}(S, U X)
$$

shows that if $\mathcal{M}$ is monogenic, then Ho $R$-Mod is also monogenic generated by $R$.

We can now complete the proof of Theorem 1.1 by noting that the natural transformation

$$
\phi_{X}: Q X \otimes_{R} G R \rightarrow G Q X=(L G)(X)
$$

is a natural transformation of coproduct-preserving exact functors on the triangulated category Ho $R$-Mod. The collection of all $X$ for which $\phi_{X}$ is an isomorphism is therefore a localizing subcategory. Since it contains $R$ and $R$ is a compact weak generator of Ho $R$-Mod, it must contain all $X \in \operatorname{Ho} R$-Mod.

The proof in the contravariant case is similar.

\section{Concluding Remarks}

The reader will note that we have actually proved the following theorem.

Theorem 2.1. Suppose $\mathcal{M}$ is a strongly cofibrantly generated symmetric monoidal model category where the unit is cofibrant. Let $R$ and $R^{\prime}$ be monoids in $\mathcal{M}$, and $G: R$-Mod $\rightarrow R^{\prime}$-Mod be a left Quillen $\mathcal{M}$-functor. Then there is a natural transformation

$$
\phi_{X}: Q X \otimes_{R} G R \rightarrow G Q X=(L G)(X)
$$

of functors on Ho $R$-Mod. Similarly, if $G: R$-Mod $\rightarrow\left(R^{\prime} \text {-Mod }\right)^{\text {op }}$ is a contravariant left Quillen $\mathcal{M}$-functor, then there is a natural transformation

$$
\psi_{X}:(L G)(X)=G Q X \rightarrow \operatorname{Hom}_{R}(Q X, F R)
$$

of functors on $\mathrm{Ho} R$-Mod.

We only used the stable and monogenic hypotheses to conclude that these natural transformations were isomorphisms for all $X$. But the natural transformations of Theorem 2.1 are natural transformations of functors that preserve homotopy colimits. So for a general $\mathcal{M}$, if $\phi_{X}$ or $\psi_{X}$ is an isomorphism for a set that of $X$ that generate Ho $R$-Mod under homotopy colimits, then $\phi_{X}$ or $\psi_{X}$ will in fact be an isomorphism for all $X$. In general, the homotopy category of any cofibrantly generated model category where the domains of the generating cofibrations are cofibrant is generated under homotopy colimits by the domains and codomains of the generating cofibrations; this is a less precise way of saying that every cofibrant 
object can be built from 0 by taking transfinite compositions of pushouts of the generating cofibrations.

We therefore have the following theorem, whose proof uses standard model category techniques but would lengthen the paper.

Theorem 2.2. Suppose that the hypotheses of Theorem 2.1 hold. In addition, suppose that one of the following conditions hold:

(1) The domains of the generating cofibrations of $\mathcal{M}$ are cofibrant and $\phi_{A \otimes R}$ (resp. $\left.\psi_{A \otimes R}\right)$ is an isomorphism for any $A$ that is a domain or codomain of one of the generating cofibrations of $\mathcal{M}$.

(2) $R^{\prime}$-Mod is left proper (resp. right proper) and $\phi_{A \otimes R}\left(\right.$ resp. $\left.\psi_{A \otimes R}\right)$ is an isomorphism for any $A$ that is a domain or codomain of one of the generating cofibrations of $\mathcal{M}$.

(3) $\mathcal{M}$ is stable and $\phi_{A \otimes R}\left(\right.$ resp. $\left.\psi_{A \otimes R}\right)$ is an isomorphism for any $A$ that is a cokernel of one of the generating cofibrations of $\mathcal{M}$.

Then $\phi_{X}\left(\right.$ resp. $\left.\psi_{X}\right)$ is an isomorphism for all $X \in \operatorname{Ho} R$-Mod.

In particular, the Eilenberg-Watts theorem holds for simplicial sets and topological spaces.

Corollary 2.3. Suppose $\mathcal{M}$ is the standard model category of simplicial sets or (compactly generated weak Hausdorff)topological spaces. Let $R$ and $R^{\prime}$ be monoids in $\mathcal{M}$, and $G: R$-Mod $\rightarrow R^{\prime}$-Mod be a left Quillen $\mathcal{M}$-functor. Then there is a natural isomorphism

$$
\phi_{X}: Q X \otimes_{R} G R \rightarrow G Q X=(L G)(X)
$$

of functors on Ho $R$-Mod. Similarly, if $G: R$-Mod $\rightarrow\left(R^{\prime} \text {-Mod }\right)^{\text {op }}$ is a contravariant left Quillen $\mathcal{M}$-functor, then there is a natural isomorphism

$$
\psi_{X}:(L G)(X)=G Q X \rightarrow \operatorname{Hom}_{R}(Q X, F R)
$$

of functors on Ho $R$-Mod.

Proof. We just do the covariant case. Any codomain $A$ of the generating cofibrations of $\mathcal{M}$ is trivially cofibrant (it is a disk), from which it follows easily that both

$$
(A \otimes R) \otimes_{R} F R \cong A \otimes G R \text { and } G(A \otimes R)
$$

are trivially cofibrant. The domains $A$ of the generating cofibrations of $\mathcal{M}$ are spheres, so the map

$$
A \otimes G R \rightarrow G(A \otimes R)
$$

is just a suspension of the weak equivalence obtained when $A=S$. The result now follows from Theorem 2.2

\section{REFERENCES}

[CKN01] J. Daniel Christensen, Bernhard Keller, and Amnon Neeman, Failure of Brown representability in derived categories, Topology 40 (2001), no. 6, 1339-1361, DOI 10.1016/S0040-9383(00)00015-X. MR.1867248(2003i:16013)

[Hir03] Philip S. Hirschhorn, Model categories and their localizations, Mathematical Surveys and Monographs, vol. 99, American Mathematical Society, Providence, RI, 2003. MR.1944041 (2003j:18018)

[Hov99] Mark Hovey, Model categories, Mathematical Surveys and Monographs, vol. 63, American Mathematical Society, Providence, RI, 1999. MR1650134 (99h:55031) 
[HSS00] Mark Hovey, Brooke Shipley, and Jeff Smith, Symmetric spectra, J. Amer. Math. Soc. 13 (2000), no. 1, 149-208, DOI 10.1090/S0894-0347-99-00320-3. MR.1695653 (2000h:55016)

[Kel94] Bernhard Keller, Deriving DG categories, Ann. Sci. École Norm. Sup. (4) 27 (1994), no. 1, 63-102. MR1258406 (95e:18010)

[SS00] Stefan Schwede and Brooke E. Shipley, Algebras and modules in monoidal model categories, Proc. London Math. Soc. (3) 80 (2000), no. 2, 491-511, DOI 10.1112/S002461150001220X. MR1734325(2001c:18006)

Department of Mathematics, Wesleyan University, Middletown, Connecticut 06459 E-mail address: mhovey@wesleyan.edu 